\title{
The effect of a combined choline salicylate and cetalkonium chloride gel on particular strains of Pseudomonas aeruginosa, Staphylococcus spp. and Streptococcus spp.
}

\author{
Saule Amangeldykyzy ${ }^{1}$, Aidana Nurlybekkyzy Nurlybek ${ }^{1}$, Aigerim Nurlybekkyzy Nurlan ${ }^{1}$, \\ Konrad T. Juszkiewicz ${ }^{2}$, Andrzej Polski ${ }^{3 \star}$, Ulzhan Baurzhankyzy Seisembay ${ }^{1}$, \\ Adiya Maksatovna Mukasheva ${ }^{1}$, Urumbaeva Kathira Umirzakovna ${ }^{1}$, Ewa Poleszak ${ }^{3}$
}

\begin{abstract}
${ }^{1}$ Department of Microbiology, Virology and Immunology, Kazakh National Medical University, 88 Tole Bi Street, Almaty, Kazakhstan ${ }^{2}$ DRK Biomedical Research and Development LLC, 788 Los Alamos avenue, Livermore, CA, USA

${ }^{3}$ Chair and Department of Applied Pharmacy, Faculty of Pharmacy, Medical University of Lublin, 1 Chodzki, 20-093 Lublin, Poland
\end{abstract}

\section{ARTICLE INFO \\ Received 25 March 2015 \\ Accepted 28 April 2015}

\section{Keywords:}

choline salicylate,

cetalkonium chloride

Pseudomonas aeruginosa,

Staphylococcus spp.,

Streptococcus spp.

\begin{abstract}
The ongoing control of virulent bacteria strains is a challenge for today's medicine. An example of this, is one widely used drug employed in treating less serious external oral and ocular bacterial infections. This is a gel containing both cetalkonium chloride and choline salicylate. However, whether in the era of expanding bacterial resistance this gel is still effective, is not clear. Hence, in our work, its antibacterial effect was studied against 13 strains of Pseudomonas aeruginosa, 6 strains of Staphylococcus spp. and 6 strains of Streptococcus spp. drawn from the collection of the Department of Microbiology, Virology and Immunology, Kazakh National Medical University, as well as against 30 strains of Staphylococcus spp. recently isolated from Kazakh medical students. This work demonstrated that Pseudomonas aeruginosa was insensitive to this preparation in all samples, while the sensitivity of Staphylococcus spp. and Streptococcus spp. was almost halved, compared to untreated samples. An interesting discovery was the greater resistance of strains obtained from student volunteers than from the collection. However, despite the evident resistance of some strains to the combined cetalkonium chloride and choline salicylate gel, we put forward that it can still be used in less serious external bacterial infections.
\end{abstract}

\section{INTRODUCTION}

Pseudomonas aeruginosa is a gram-negative, aerobic, citrate, catalase and oxidase positive bacteria, which can induce disease in humans, especially in damaged tissues or under conditions of reduced immunity. It is classified as a opportunistic pathogen, and it is widespread in the world, as human skin flora, in water, etc, yet, it can be a health danger, even inducing death, particularly on having colonized the lungs, kidneys or other vital organs. More often, however, it brings about general inflammation, and only rarely sepsis [3]. Staphylococcus spp. strains are gram-positive bacteria, not always pathogenic, mainly found on human skin and in the respiratory tract. They induce skin infections and respiratory disease. Staphylococcus aureus is the most famous

\footnotetext{
* Corresponding author

e-mail: andrzejpolski@umlub.pl

tel.: +48 8144870 40, fax: +48 814487040
}

representative of this group [5,8]. Methicillin-resistant Staphylococcus aureus (MRSA) is resistant to the majority of $\beta$-lactam antibiotics, and brings with it, many problems in patient treatment [18]. Streptococcus spp. are also grampositive bacteria strains, not all of which are pathogenic. While they are part of the normal human flora, some of them induce such dangerous diseases as: conjunctivitis, meningitis, flesh-eating disease, endocarditis, etc [14].

Inflammatory disease (inflammation) is the term utilized for the complex response of an organism to certain harmful factors. Among these are bacteria, damaged cells, etc [6]. It involves specific enzyme activation, the release of cytokines, the migration of cells, and, eventually, it leads to damage repair [17].

Choline salicylate (Fig. 1) an anti-inflammatory drug, similar to its most famous derivative - acetylsalicylic acid. It is commonly used in the treatment of pain, fever and 
rheumatic disorders. The standard dose of $325 \mathrm{mg}$ acetylsalicylic acid is equal to about $435 \mathrm{mg}$ of choline salicylate. In the treatment of pain and fever, it is used orally in 435-870 mg doses administered every few hours, or in divided daily doses of 4.8-7.2 $\mathrm{g}$ for rheumatic disorders. As a $20 \%$ solution, it is used as a local analgesic in ear disorders and to soften ear wax for its further removal. In the form of an $8.7 \%$ gel, it is also sometimes employed for treating lesions of the mouth. In addition, Choline salicylate is used topically for muscular and rheumatic pain [11]. The best known side effect of choline salicylate is that shared with acetylic acid. This is Reye's syndrome. Hence, when administered to children, it should be used carefully [13]. Cetalkonium chloride (Fig. 2) is an ammonium antiseptic agent (a derivative of benzalkonium chloride) used in many topical drugs for infections of mouth, throat and eye [10]. Its activity is based on the creation of a positive charge, which gives it bio-adhesive properties with regard to negative charges on surfaces. It is rarely used in concentrations more than 5\% $[4,9]$.

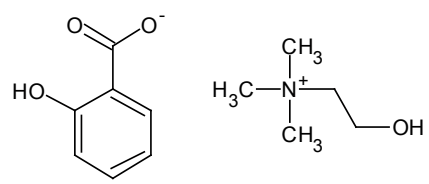

Figure 1. Choline salicylate structure

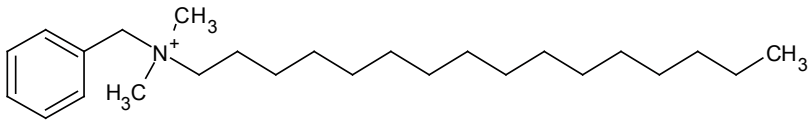

$\mathrm{Cl}^{-}$

Figure 2. Cetalkonium chloride structure

The main aim of this study was to determine the sensitivity of Pseudomonas aeruginosa, Staphylococcus spp. and Streptococcus spp., both archive and patient strains, to a combined choline salicylate and cetalkonium chloride gel. In our work, we utilized samples of 13 strains of Pseudomonas aeruginosa, 6 strains of Staphylococcus spp. and 6 strains of Streptococcus spp. These were obtained from the collection of the Department of Microbiology, Virology and Immunology, Kazakh National Medical University. In addition, we used samples of 30 strains of Staphylococcus spp. isolated recently from the noses and throats of volunteer Kazakh medical students.

\section{MATERIALS AND METHODS}

This paper examined a topical medication in a form of a gel containing two active substances (choline salicylate and cetalkonium chloride). This gel included as excipients: hydroxypropyl methylcellulose, glycerol, sodium cyclamate, anise essential oil, menthol, ethanol, and purified water.

In our work, the biological biochemical and morphological properties of selected archive strains were evaluated. Pseudomonas aeruginosa are motile, gram (-) rod-shaped bacteria that do not form spores and capsules, and which can be grown on meat-peptone agar, as well as meat-broth Peptone. These bacteria induce the fermentation of glucose and the liquefaction of gelatin. Moreover, they bring about hemolysis. Pseudomonas aeruginosa spp. can be considered to be a positive oxidase. They have green fluorescence (pigment - pyocyanin). Staphylococcus spp. are gram $(+)$ cocci bacteria, that show an arrangement similar to a bunch of grapes. These bacteria do not form spores and capsules, and they are capable of isolating lecithinase and hemolysin. Staphylococcus spp. grow on meat-peptone agar, meat-broth Petone, yolk-salt agar and blood agar. Streptococcus spp. are gram $(+)$ cocci arranged bacteria that resemble a chain. They demonstrate $\beta$-hemolysis on blood agar. What is more, they are capable of growing on sugar broth as a base and as a wall surface sediment.

Bacterial growth inhibition in our study was determined by disk diffusion antibiotic sensitivity testing (A) and by way of a spread plate (B). The bacterial sensitivity was studied through employing the disk diffusion method, on the surface of yolk-salt agar, blood agar and meat-peptone agar, in Petri dishes. In this part of the study, the bacterial suspension was spread upon the base agar, and then disks were placed upon this that contain a defined concentration of the gel. The plates were then incubated at $37^{\circ} \mathrm{C}$, and after a day, the results were determined by measurement of growth (in millimeters) from the disk centre (B). The sensitivity was, as well, determined by a second method. This was carried out as follows: with a Pasteur pipette, holes were first made in plates of meat-peptone agar, at equal distances from each other, and then these holes were filled with the studied gel. Subsequently, the plates were incubated in an incubator, at $37^{\circ} \mathrm{C}$, for a one day. The diameters of the resulting zones were then measured as evidence of the antibacterial effect of the gel.

In the assessment, the degree of sensitivity was:

1) insensitive (growth inhibition $>15 \mathrm{~mm}$ ),

2) sensitive (growth inhibition $=15-25 \mathrm{~mm}$ ),

3) highly sensitive (growth inhibition $>25 \mathrm{~mm}$ ).

\section{RESULTS}

The results (Tab. 1) of bacterial growth inhibition due to the application of a combined choline salicylate and cetalkonium chloride gel, as measured by the two above methods, are as follows. Firstly, all Pseudomonas aeruginosa strains were insensitive in all samples. In the case of Staphylococcus spp. strains drawn from the collection - 9 were insensitive, 14 were sensitive and 7 were highly sensitive, while among Staphylococcus spp. strains isolated from the noses and throats of volunteer students - 20 were insensitive, 9 were sensitive and 1 was highly sensitive. An interesting situation occurred in the case of the Streptococcus spp. Here, one half of the strains were insensitive, while the second half were sensitive. Figure 3 reveals the lack of inhibition effect of this gel on strains of Pseudomonas aeruginosa drawn from the collection. Figure 4 shows the plate growth inhibition effect of this gel on a strain of Staphylococcus spp. isolated from a Kazakh medical student. Figure 5 shows the lack of growth inhibition of the gel on the strains of Streptococcus drawn from the collection. 
Table 1. Sensitivity of different bacteria on gel with Choline salicylate and Cetalkonium chloride

\begin{tabular}{|l|c|c|c|}
\hline \multirow{2}{*}{ Strain } & \multicolumn{3}{|c|}{ Sensitivity } \\
\cline { 2 - 4 } & insensitive & sensitive & $\begin{array}{c}\text { highly } \\
\text { sensitive }\end{array}$ \\
\hline Pseudomonas aeruginosa (museum) & $100.0 \%$ & - & - \\
\hline Staphylococcus spp. (museum) & $30.0 \%$ & $46.7 \%$ & $23.3 \%$ \\
\hline Streptococcus spp. (museum) & $50.0 \%$ & $50.0 \%$ & - \\
\hline Staphylococcus spp. (students) & $66.7 \%$ & $30.0 \%$ & $3.3 \%$ \\
\hline
\end{tabular}

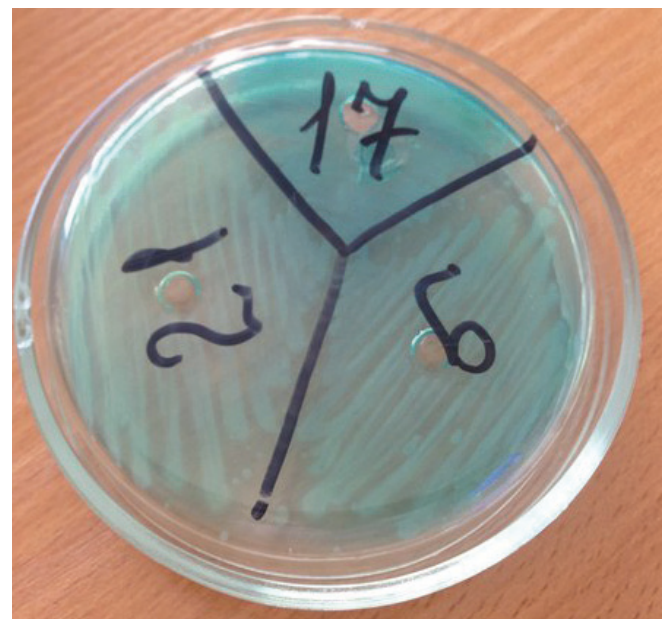

Figure 3. Pseudomonas aeruginosa on Petri dishes

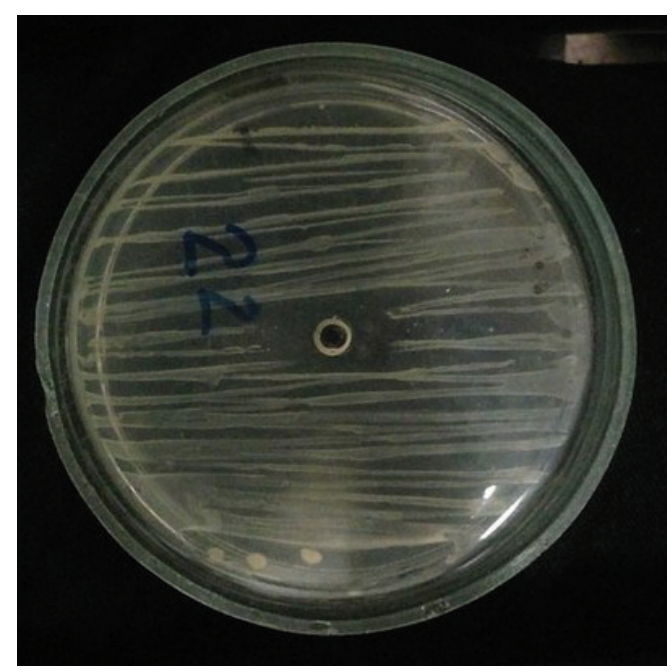

Figure 4. Staphylococcus spp. on Petri dishes

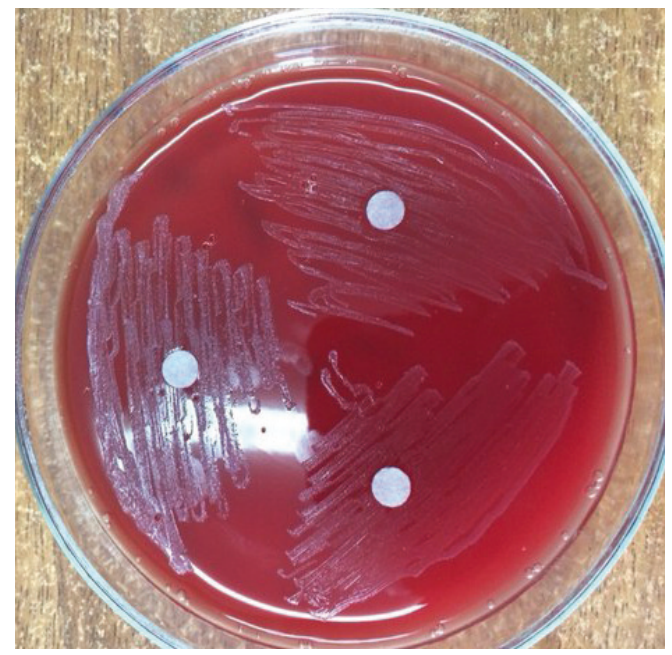

Figure 5. Streptococcus spp. on Petri dishes

\section{DISCUSSION}

The results clearly show that the gel had no effect on Pseudomonas aeruginosa (100\% insensitivity). A similar finding is described by Harrison et al. [7], wherein Pseudomonas aeruginosa was found to be insensitive to benzalkonium chloride, cetalkonium chloride, cetylpyridinium chloride, myristalkonium chloride, and Polycide, but was seen to be sensitive to all of these substances when in a mixture with added $\mathrm{Cu}^{2+}$ ions. In the case of the studied gel, only choline salicylate and cetalkonium chloride were present, as the gel was without free $\mathrm{Cu}^{2+}$ ions. The work of Bengani and Chauhan [4] provides confirmation that cetalkonium chloride (which they used as a element within an experimental mixture) can be successfully employed as antibacterial drug for ophthalmic conditions. However, the research undertaken by Tarbox et al. [16] shows that $0.1 \%$ benzalkonium chloride solution has better effect than saline against Pseudomonas aeruginosa that was found to be present in contaminated wounds.

In our work, we noted differences between the sensitivity results of Staphylococcus spp. archive strains, and those isolated from student volunteers. In first case, $30 \%$ of all drawn strains were insensitive to the gel, while in the second, almost $67 \%$ of the obtained strains showed insensitivity. Similar results were seen in the case of its sensitivity to the gel (46.7\% and 30\%, respectively). Again, a significant difference was observed in the highly sensitive strains (23.3\% and 3.3\%, respectively). These differences are most likely due to the large variety of strains present in the samples that were obtained from the student volunteers, and that such strains have resistance to cetalkonium chloride.

Benzalkonium chloride is commonly used in hospitals, as it is considered to be an effective disinfectant against many bacteria e.g. Staphylococcus spp. However, in recent times, a serious threat has appeared in the form of MRSA resistant to benzalkonium chloride [1,2]. Research carried out by Raggi et al. [15] confirm that some Staphylococcus spp. are insensitive to benzalkonium chloride. Regarding this, they claim that it is the presence of qacA/B, but not smr genes that is responsible for the demonstrated higher resistance of staphylococci to benzalkonium chloride. Finally, our work reveals that half of the Streptococcus spp. are insensitive to the studied gel, while none of these strains are highly sensitive. Our results were confirmed by those of Mosca et al. [12], who noted that benzalkonium chloride is effective against Streptococcus agalactiae.

\section{CONCLUSIONS}

Our study results are consistent with the known literature. Pseudomonas aeruginosa in most cases, is resistant to cetalkonium chloride and to other ammonium antiseptic agents. The situation, however, is found to be more complicated when it comes to Staphylococcus spp. and Streptococcus spp. Their sensitivity to ammonium antiseptic agents is individual with regard to each strain. Some are resistant, while others are sensitive. Such differences are confirmed by the dissimilarity of the sensitivity tests carried out on Staphylococcus spp. taken from student volunteers, when 
compared with archive strains. Strains living currently, can easily mutate, so they can be more resistant than archived strains. Yet, despite the resistance of many strains, the combined cetalkonium chloride and choline salicylate gel can still be used as an alternative in less serious external bacterial infections.

\section{REFERENCES}

1. Akimitsu N., Hamamoto H., Inoue R.: Increase in Resistance of Methicillin-Resistant Staphylococcus aureus to $\beta$-Lactams Caused by Mutations Conferring Resistance to Benzalkonium Chloride, a Disinfectant Widely Used in Hospitals. Antimicrob. Agents Chemother., 43, 3042, 1999.

2. Al-Masaudi S.B., Day M. J., Russell A. D.: Sensitivity of methicillinresistant Staphylococcus aureus strains to some antibiotics, antiseptics and disinfectants. J. Appl. Bacteriol., 65, 330, 1988.

3. Balcht A., Smith R.: Pseudomonas aeruginosa: Infections and Treatment. Informa. Healthcare., 2, 83, 1994.

4. Bengani L.C., Chauhan A.: Extended delivery of an anionic drug by contact lens loaded with a cationic surfactant. Biomaterials, 34, $2817,2013$.

5. Cole A.M. et al.: Determinants of Staphylococcus aureus nasal carriage. Clin. Diagn. Lab. Immunol., 8, 1066, 2001.

6. Ferrero-Miliani L. et al.: Chronic inflammation: importance of NOD2 and NALP3 in interleukin-1beta generation. Clin. Exp. Immunol., 147, 227, 2007.

7. Harrison J.J. et al.: Copper and Quaternary Ammonium Cations Exert Synergistic Bactericidal and Antibiofilm Activity against Pseudomonas aeruginosa. Antimicrob. Agents Chemother., 52, 2874, 2008.
8. Kluytmans J. et al.: Nasal carriage of Staphylococcus aureus: epidemiology, underlying mechanisms, and associated risks. Clin. Microbiol. Rev., 10, 508, 1997.

9. Lallemand F. et al.: Successfully improving ocular drug delivery using the cationic nanoemulsion, novasorb. J. Drug Deliv., 604204, 3,2012 .

10. Martindale: The Complete Drug Reference. [online] London: Pharmaceutical Press. http://www.medicinescomplete.com (cited: 07.01.2015).

11. Micromedex 2.0, (electronic version). Truven Health Analytics, Greenwood Village, Colorado, USA. Available at: http://www. micromedexsolutions.com/ (cited: 12.01.2015).

12. Mosca F., Russo F., Miragliotta G.: (2006) In vitro antimicrobial activity of benzalkonium chloride against clinical isolates of Streptococcus agalactiae. J. Antimicrob. Chemother., 57, 566, 2006.

13. Oman T. K. et al.: Topical choline salicylates implicated in Reye's syndrome. BMJ, 336, 1376, 2008.

14. Patterson M.J. (1996). Streptococcus. In: Baron's Medical Microbiology (Baron S. et al., eds.) (4th ed.). Univ of Texas Medical Branch.

15. Raggi C., et al.: Methicillin Resistance, Biofilm Formation and Resistance to Benzalkonium Chloride in Staphylococcus aureus Clinical Isolates. Clin. Microbial., 2, 3, 2013.

16. Tarbox B.B. et al.: Benzalkonium chloride. A potential disinfecting irrigation solution for orthopaedic wounds. Clin Orthop Relat Res., 346, 257, 1998.

17. Vane J.R., Botting R.M.: New insight into the mode of action of ant-inflammatory drugs. Inflamm. Res., 44, 2, 1995.

18. Waters A.E. et al.: Multidrug-Resistant Staphylococcus aureus in US Meat and Poultry. Clin. Infect. Dis., 52, 1227, 2011. 